\title{
EL ESCÁNDALO DE LA CELESTINA: MAGIA Y ACUMULACIÓN PRIMITIVA EN LA ESPAÑA DEL HOLOCAUSTO (1486-1507) ${ }^{1}$
}

\author{
Víctor M. Pueyo Zoco \\ Temple University - Philadelphia, PA, EE.UU. \\ vpueyozo@temple.edu
}

$\mathrm{N}$ o es que la cuestión de la magia haya ocupado un lugar marginal en la bibliografía celestinesca. Atrás quedan, en la distancia, trabajos importantes como los de Patrizia Botta (1994) y Dorothy Severin $(1993 ; 1995)$ en los años noventa, que encontrarían un amplio eco en los de las generaciones posteriores. Una parte no despreciable de ellos indaga vestigios de textos y autores de la biblioteca clásica en la redacción del conjuro, como Lucano (Severin 2006), Apuleyo y Petronio (Padilla Carmona 2017: 236) o san Justino y san Cipriano (Lozano Renieblas 2005: 153-64); otros libran una acalorada querella sobre la veracidad del conjuro, que enfrenta a quienes señalan el carácter artificiosamente literario del texto (Pérez Priego 2000) contra los que prefieren subrayar sus afinidades con auténticos sortilegios medievales (Russell 1978: 241-76), como los que combate el libro V del famoso Formicarius de Johannes Nider (Lara Alberola y Montaner 2016: 447-449). La duda no es baladí, puesto que en ella podría cifrarse la diferencia entre el pasatiempo lúdico de una mera hechicera y un verdadero acto de brujería. Finalmente, hay contribuciones que van más allá del análisis de este conjuro en sí o de sus fuentes remotas. Me refiero a las que ponen su acento en la reivindicación del libre albedrío de Melibea (Cárdenas Rotunno 2001: 371374; López Griguera 2005: 111-24; Canet 2000: 201-211) frente a las que insisten

$1 \quad$ Este artículo se ha desarrollado dentro del proyecto «La mujer frente a la Inquisición española y novohispana» (FEM2016-78192-P), I+D de Excelencia del Ministerio de Economía y Competitividad (MINECO), financiado por la Agencia Estatal de Investigación (AEI) y el Fondo Europeo de Desarrollo Regional (FEDER, UE); y del grupo de investigación «Mentalidades mágicas y discursos antisupersticiosos (siglos XVI, XVII y XVIII)», grupo consolidado por la Universidad Autónoma de Madrid.

Edad de Oro, XXXVIII (2019), pp. 35-53, ISSN: 0212-0429 - ISSNe: 2605-3314

DOI http://doi.org/10.15366/edadoro2019.38.002 
en la efectividad de la philocaptio urdida por Celestina (Severin 1995; Dangler 2001: 19-50); guerra abierta de posiciones que no deja de plantear una suerte de dilema para el feminismo liberal: si el conjuro surte efecto y condiciona el comportamiento de Melibea, eso limita su capacidad de amar libremente, pero, si no es así, la mediación de Celestina se torna irrelevante y, con ella, también sus mañas profesionales como curandera y cocinera de afrodisíacos, que Rojas trataría de descalificar asociándolas a la brujería.

Los debates son tan inagotables como la bibliografía misma. Pero la pregunta de fondo, la que en todo momento debió protagonizar el estado de la cuestión, sigue sin ser abordada de manera frontal en ninguno de estos trabajos. La tragicomedia de Calisto y Melibea, el libro que con el paso de los siglos hemos acabado conociendo como La Celestina, es ante todo un libro sobre una mujer con fama de hechicera que vive o malvive en la Europa de finales del siglo xv, mientras cientos de mujeres están siendo arrojadas a la hoguera bajo la sospecha de ser brujas, esto es, acusadas de cometer crímenes que son (no solo, pero, sobre todo) crímenes femeninos. Y esta situación, la situación en la que Celestina existe, la situación alrededor de la cual irremisiblemente y para siempre pivota la trama de la tragicomedia, es una situación que permanece ausente del grueso de los debates sobre la obra, por lo menos en tanto permanecen ausentes también términos como «feminicidio», «acumulación primitiva» o «cercamiento y privatización de los bienes comunes». Esta es la situación a la que me quiero referir en lo sucesivo como el escándalo de La Celestina.

Hechicera o bruja, papagayo de una longeva tradición literaria o verdadera «cliéntula» del demonio, Celestina comparece en este extraño drama cortesano y su presencia es en sí misma una anomalía que demanda una explicación. ¿En qué se basa el triunfo del relato de una hechicera en 1499 si la brujería, como afirma Severin, «was not a particularly hot topic in Spain at the end of the fifteenth century» (1993: 11)? Y más aún: ¿por qué ocupa la hechicera/medianera el centro del relato, «muy alejada» — como bien recuerda Padilla Carmona - «de su papel secundario en los antecedentes clásicos que venían desde la comedia griega» (2017: 231)? Estas son las preguntas que pretendo contestar en lo sucesivo. Después de sortear todos los obstáculos exegéticos, tras recorrer los múltiples meandros que nos llevan del nacimiento a la desembocadura del texto, uno sigue teniendo la sensación de que algo se nos sigue escapando entre los dedos, de que hay un elefante en la habitación de la crítica celestinesca y de que ese elefante es —y sigue siendo - Celestina.

\section{La dialéctica en el burdel: de Marx a Federici (y de Federici a Marx)}

Quizá lo más curioso de estas preguntas es que ya han sido contestadas. En su influyente Calibán y la bruja, publicado originalmente en 1998, Silvia Federici 
lanzaba una provocativa tesis que todavía está por ponerse a prueba en el ámbito de los estudios celestinescos. Esta tesis podría formularse de manera muy simple así: el capitalismo no es, contra lo que el sentido común dialéctico parece dictar, lo opuesto al feudalismo; el capitalismo temprano (el primer mercantilismo europeo) es, políticamente hablando, el nombre de la contrarrevolución feudal encargada de reprimir las luchas anti-feudales que se habían desarrollado desde al menos mediados del siglo XIV, especialmente desde que empezara a recrudecerse el proceso de enclosure o cercamiento de tierra arable en toda Europa. Es decir, no es que las relaciones de producción capitalistas vinieran a negar las estructuras feudales, sino que lo que negaban era esa negación previa o negación interna (el cuarto término de la dialéctica) que Fredric Jameson (1973) y Slavoj Žižek (2008: 180-182) llamaron mediación evanescente (Pueyo 2012). Esto afecta de manera crucial a la situación de las mujeres en la transición al modo de producción capitalista. El cándido «pasar página» de la historiografía liberal (con su imagen del individuo «libre» que se afirma contra los obstáculos del feudalismo y los niega o reniega de ellos) implica que el patriarcado es una especie de residuo que se cura con más liberalismo. Federici, en cambio, contiende que el patriarcado no es a finales del siglo Xv un residuo feudal, sino el resultado del largo proceso de cercamiento que privatiza los espacios y quehaceres que solían ser comunes en las zonas rurales:

En la aldea feudal no existía una separación social entre la producción de bienes y la reproducción de la fuerza de trabajo; todo el trabajo contribuía al sustento familiar. Las mujeres trabajaban en los campos, además de criar a los niños, cocinar, lavar, hilar y mantener el huerto; sus actividades domésticas no estaban devaluadas y no suponían relaciones sociales diferentes a las de los hombres, tal y como ocurriría luego en la economía monetaria, cuando el trabajo doméstico dejó de ser visto como trabajo real. Si tenemos también en consideración que en la sociedad medieval las relaciones colectivas prevalecían sobre las familiares, y que la mayoría de las tareas realizadas por las siervas (lavar, hilar, cosechar y cuidar los animales en los campos comunes) eran realizadas en cooperación con otras mujeres, nos damos cuenta de que la división sexual del trabajo, lejos de ser una fuente de aislamiento, constituía una fuente de poder y de protección para las mujeres. Era la base de una intensa sociabilidad y solidaridad femenina que permitía a las mujeres plantarse en firme ante los hombres, a pesar de que la Iglesia predicase sumisión y la Ley Canónica santificara el derecho del marido a golpear a su esposa (Federici 2010: 40-41).

La fractura entre la esfera de la producción y la de la reproducción se gesta de hecho en el interior de una lógica ya plenamente capitalista, que distingue entre trabajo asalariado del que no lo es y que privilegia el primero. El trabajo reproductivo, que dependía en gran medida de las mujeres en la aldea feudal, se categoriza como no asalariado y permanece fuera del tráfico de los tratos y contratos que propician la creciente proletarización de las masas campesinas en el escenario 
urbano. Muchas mujeres como Celestina o como Lozana advertirían esta brecha y no tendrían más remedio que buscarse la vida en la ciudad participando de los mercados informales que quedan fuera del más formal o mercado propiamente dicho. Incapaces de vender su fuerza de trabajo como mujeres, venden su cuerpo o los cuerpos de otras mujeres (Elicia, Areúsa) y se transforman en prostitutas o medianeras para rellenar el vacío dejado por la desvalorización del trabajo reproductivo en el naciente mundo capitalista. Esta es precisamente la justa crítica que Federici dirige a Karl Marx desde un feminismo marxista: su olvido del papel que la desvalorización del trabajo reproductivo jugó en el proceso de acumulación primitiva que cimentó el actual régimen de desigualdad ya entre los siglos XV y XVI.

En el primer libro de El capital, Marx, recordemos, carga en la cuenta de este proceso a dos víctimas. Por un lado, señala a los campesinos expulsados de sus tierras por las compras, subastas públicas y anexiones ilegales de tierras del procomún, primero de manera ilegal y luego ya (a partir de 1604 en Inglaterra, algo más tarde en España) bajo el amparo de la ley. Estos, a veces jornaleros, pero casi siempre pequeños propietarios, engrosarán el nuevo contingente de trabajadores desterrados y sin tierra que Marx llamó proletariado (2007: 784-849). España estuvo lejos de ser una excepción a esta lenta dinámica de apropiación. En Andalucía, por ejemplo, se fijan desde la Reconquista dos tipos de propiedad de la tierra: la privada y la comunal, a la que pertenecen los bienes concejiles. Estos, a su vez, se subdividen en «bienes de propios, cuya explotación iba en beneficio del concejo y bienes comunales de cuyo provecho se servían todos los vecinos de la comunidad» (Carmona Ruiz 1995: 54). Los bienes de propios son, tal y como los definen las Siete Partidas, aquellos bienes inapropiables «que pertenescen al pro comunal de toda la cibdad o villa» (Alfonso X «el Sabio» 1807: 713). Los bienes comunales, también detallados en el código de Alfonso X, son por contra aquellos que, no pudiéndose sujetar a la propiedad privada, tampoco generan renta en pro del concejo, pudiendo ser libremente aprovechados por todos los habitantes de la villa. Aquí habría que contar las llamadas tierras baldías o realengas, tierras que no estaban destinadas al cultivo, pero cuya explotación se consideraba de derecho común, ya fueran utilizadas para el ganado o para la obtención de materias primas necesarias para el sostenimiento diario (Carmona Ruiz 1995: 61).

La usurpación sistemática de estas tierras comienza en el siglo xIV y se intensifica a lo largo del Xv. En el caso de Andalucía, lo hace con la apropiación de tierras concejiles, las ampliaciones ilegales de tierras de la dehesa y la eliminación de distintos derechos comunales, como el pastoreo común del ganado. Al mismo tiempo, los terrenos baldíos o de uso comunal fueron monopolizados, «no solo por los vecinos del común, sino por la nobleza y los oficiales del concejo, quienes aumentaron sus posesiones a costa de las tierras de titularidad pública» (Carmona Ruiz 1995: 81). No obstante, las principales usurpaciones fueron realizadas 
por los miembros de la oligarquía local, escudados en el poder político que ostentaban. Las Cortes de Toledo de 1480 intentaron restituir los terrenos y bienes usurpados, pero las audiencias se dilataban constantemente y las campañas de la Guerra de Granada dificultaron todavía más una reposición inmediata (Carmona Ruiz 1995: 96). ¿Era esta una situación exclusiva de Andalucía? En modo alguno. Alberto Marcos Martín analiza la apropiación de tierras y bienes comunes en el espacio de la cuenca del Duero (actual Castilla y León). En su estudio se refiere a los bienes de propios y comunes como propiedad municipal, en tanto señalan «una capacidad de gestión y usufructo común» (Marcos Martín 1997: 59). Como Carmona Ruiz, identifica la usurpación de tales bienes, entre los siglos XIV y XV por parte de la oligarquía nobiliaria, que continuaron y aun se incrementaron a lo largo del seiscientos. Llama la atención sobre la intervención de los Reyes Católicos en las Cortes de Toledo, para agregar que fue también la Corona la que promovió «estos abusos o al menos creó las condiciones necesarias para que pudieran producirse» (Marcos Martín 1997: 72)2.

Por supuesto, la acumulación primitiva de tierras y plusvalías no dependía solamente de la expulsión del campesino del procomún, pariente cercano y condición de posibilidad del pícaro. Marx cargaría también en su cuenta el sacrificio de varios millones de cuerpos indígenas en el contexto del mayor cercamiento de suelo virgen conocido por la humanidad, donde a comienzos del siglo XVII los españoles se habían apropiado de aproximadamente un tercio de las tierras comunales indígenas bajo el sistema de la encomienda (que es, entre otras cosas, un tipo concreto de cercado ratificado por las Leyes de Burgos de 1512). Pero Marx, denuncia Federici, obviaba una tercera víctima propiciatoria que suele ocupar en los textos literarios de la época el lugar de la tercería y cuyo análisis resulta inexcusable cuando hablamos de La Celestina. La venta de la fuerza de trabajo explica los trazos más gruesos del desarrollo capitalista en su primera fase mercantil, pero no acaba de poner en valor el «trabajo reproductivo o trabajo pasivo»; su dimensión, digamos, «biopolítica». Este es un trabajo que escapa a la retribución salarial y que reclama su propia literatura, donde «el cuerpo es para las mujeres lo que la fábrica es para los trabajadores asalariados varones: el principal terreno de su explotación y resistencia» (Federici 2010: 29). No me parece en absoluto arriesgado postular que La Celestina misma es el primer y el mejor exponente de aquella literatura, que conocerá una feroz y feraz progenie.

2 Además de estas usurpaciones en España, José Luis Martín Martín (1990: 33-35) se refiere a otros tipos de «utilización privativa», que incluyen «la particularización de uso», es decir, «la decisión concejil de suspender el aprovechamiento colectivo de determinados bienes para entregarlos a un particular», así como «la privatización absoluta de adquisición de la propiedad del antiguo bien común por una sola persona o familia» (33-34). Igualmente, destaca la apropiación de las rentas por parte los mayordomos y arrendadores al negarse a devolver lo pactado para usos comunes. 
Esto ilustra, ciertamente, el estatuto de Celestina como medianera, madama o sultana del lenocinio, traficante de virgos, etc. Pero ¿qué tiene que ver con la brujería? Naturalmente, todo. Cuando estas mujeres traten de recuperar las prestaciones del valor perdido a través de una gestión del cuerpo en la esfera pública (prestaciones que sí obtenían cuando el trabajo reproductivo era comunitario), el desempeño de este trabajo reproductivo no asalariado va a contemplarse alternativamente de dos maneras: a) desde el punto de vista de la nueva ideología mercantilista, se trata de un trabajo improductivo que debe realizarse en la esfera privada de la domesticidad, cuyos contornos ahora se definen por contraposición al ámbito público del trabajo; y b) desde el prisma de la vieja ideología feudalizante, este trabajo es practicado en un lugar fuera de lugar, es decir, fuera del orden de signaturas providencialmente impuesto para seguir garantizando la lectura del mundo; esto lo convierte, automáticamente, en un trabajo diabólico. De esta manera, y en el trenzado ideológico que establece la problemática de la transición, la dejación de las funciones domésticas en la esfera privada no tarda en granjearse acusaciones de brujería. Es por esta razón que las brujas son representadas cabalgando sobre una escoba ya en textos misóginos franceses como Le champion des dames (escrito en 1451, pero impreso en 1485) y, por ello, que algunos de los cargos criminales más frecuentes que pesan sobre estas «brujas» (a menudo retratadas como mujeres ajadas de úteros yermos, e.g., nuestra «puta vieja Celestina») son la ingesta de niños y su capacidad de provocar la impotencia en los hombres ${ }^{3}$. Sobra decir que la supuesta improductividad — i.e., esterilidad — del trabajo reproductivo no asalariado se manifiesta también en la continua condena del mercado del sexo, que Celestina, madre-que-no-es-madre, representa sobradamente, así como en la tendencia a la masculinización de la bruja, que explica la alusión a Celestina como una «vieja barbuda» ${ }^{4}$. Mención dilatada, pero no aparte, merecerían todos aquellos conjuros o cantrips que escenifican la comisión mágica de tareas domésticas — platos que se lavan solos, menús que prepara una mano invisible como la de Adam Smith - y que sobreviven todavía vinculados a la brujería en

3 Lo primero, todavía en textos como el Compendium maleficarum de Francesco Guazzo (1608). Ver Martin Le Franc (1485: f. 105v) y Guazzo (2012: 33). La relación entre magia e impotencia goza de una no menos fecunda suerte. Catherine Rider (2006) advierte de su proliferación en los tratados y debates médicos del siglo xv. El propio Malleus maleficarum había estado «particularly interested in the relationship between witchcraft and sex, but the notion that witches attacked fertility seems to have been widespread, and it fitted in well with the idea that witches attacked whatever was central to Christian society» (Institoris y Sprenger 2006: 187).

4 No hay que olvidar que las parteras, como participantes de un parto que no es el suyo, son también el blanco de estos libelos, empezando por la obra de Heinrich (Kramer) Institoris y Jakob Sprenger: «No one does more harm to the Catholic Faith than midwives. For when they do not kill children, then, as if for some other purpose, they take them out of the room and, raising them up in the air, offer them to devils» (1970: 66). 
las películas de la factoría Disney. Pero erraríamos el tiro si limitáramos el alcance de las acusaciones de brujería al desempeño de o a la falta de empeño en quehaceres domésticos. No se plantea exactamente que mujeres tocadas por la varita de un súbito liberalismo desearan zafarse de estos quehaceres e incorporarse a un nuevo mercado de trabajo ciudadano, que sería tan poco halagüeño para ellas como para sus homólogos masculinos. De hecho, algunas sí lograban hacerlo, con varia suerte, mientras que la mayoría simplemente trataba de seguir ejerciendo precariamente aquel trabajo reproductivo que otrora conviviera de manera indistinta con el productivo en tanto del procomún

Celestina misma combina varios de estos empleos para ganarse la vida, tal y como refrenda un famoso pasaje en boca de Pármeno: «Ella tenía seis oficios, conviene a saber: labrandera, perfumera, maestra de hacer afeites y de hacer virgos, alcahueta y un poquito de hechicera» (Rojas 2011: 54). Lucrecia amplía la nómina de ocupaciones a más de treinta: «perfuma tocas, hace solimán, y otros treinta oficios; conoce mucho en yerbas, cura niños, y aun algunos la llaman la vieja lapidaria» (Rojas 2011: 115). Y a este prolífico currículum habría todavía que añadir el trabajo de partera, que la propia Celestina nos revela cuando menciona que Calisto tiene veintitrés años, pues «le vido nacer y le tomó a los pies de su madre» (Rojas 2011: 133).

El problema no es, por tanto, el acceso de la mujer a un salvífico mercado (tesis que podría guarecerse en el feminismo liberal de Severin y en su tesis, parcialmente cierta, del «empoderamiento» de las mujeres), ni siquiera la mercantilización en sí de oficios que se consideraban femeninos y que podrían haber conservado su valor en el mercado; el problema es la devaluación y el progresivo cercamiento del legado de saberes y quehaceres que beneficiaban a la comunidad y que sobrevivían agazapados en el estómago del mundo estamental. El prestigio de estos saberes, que después en efecto será parcialmente redimido por el patriarcado mediante su reglamentación y su profesionalización, no debe subestimarse. Todavía Melibea llama a Celestina «mujer bien sabia y maestra grande» (Rojas 2011: 223), en una apreciación — valorización del trabajo, al fin y al cabo- que sería demasiado fácil atribuir a la ingenuidad del personaje o a la ironía del autor ${ }^{6}$.

$5 \quad$ La incorporación de las mujeres a la disciplina del mercado de trabajo durante la Baja Edad Media es un aspecto sobradamente estudiado y documentado en ordenamientos de cortes, fueros municipales, registros mercantiles y burocracia testamentaria. Destaca su participación en el sector artesanal y textil (las sederas), en la hostelería, en la medicina, en el comercio e incluso en las finanzas (Herrero y Pérez Galán 2014; Solomon 1997; Muñoz Fernández y Segura 1988).

6 No es la única vez que Melibea o Pármeno elogian las destrezas de Celestina. Brinkop nos recuerda que estas «brujas» eran en realidad «mujeres sabias» a las que recurrían «tanto campesinos como citadinos en busca de remedios contra enfermedades e infortunios, filtros para el amor y contra la impotencia, ayuda durante los embarazos difíciles y asistencia en los partos» (2014: 42). 


\section{Volver a La CELESTINA}

Solo el análisis de esta fractura de las lógicas comunitarias del modo de producción feudal puede en consecuencia evidenciar la clave de aquello sobre lo que $L a$ Celestina inevitablemente tiene que hablar (incluso si Rojas quisiera abordar otra cosa), que es asimismo la piedra de toque de todo examen serio sobre el fenómeno de la brujería en las formaciones sociales de transición. Al que piense que esta interpretación es exagerada, que la brujería tiene que ver simplemente con la brujería y no con la problemática histórica que se trata de desplegar aquí, habría que remitirle a la intención del autor, que acaso no sea la última palabra sobre la obra, pero sí un testimonio sintomático más y nunca el menos importante. La intención, claramente expresada en la «carta del autor a un su amigo», es reprender a los amantes y sus mentidas razones de amor, no menos que avisar contra los engaños de las alcahuetas y de las «falsas mujeres hechiceras» (Rojas 2011: 6). Esta expresión no debería llamarnos la atención. Rojas podría haberse referido a las falsas hechiceras, lo que sin duda ratificaría el énfasis antes referido en el libre albedrío de Melibea, pero dice «falsas mujeres» (hechiceras), lo que no apunta tanto a la falsedad de las mujeres como hechiceras cuanto, vertiginosamente, a la falsedad de las hechiceras como mujeres?

Tal vez haya que recordar que la palabra «aquelarre» (del vasco akelarre, «prado del macho cabrío») reúne en una misma unidad de significado dos semantemas que parecen necesitarse mutuamente para que la palabra tenga sentido: aquelarre significa reunión o tumulto de brujas y aquelarre es el acto mismo por el que dichas brujas invocan al demonio. Federici nota «que el pacto entre la bruja y el Diablo era llamado conjuratio, como los pactos que perpetraban frecuentemente los esclavos y los trabajadores en lucha» (2010: 243) a lo largo de la Antigüedad. Pero no hacía falta remontarse al imperio romano para descubrir este solapamiento entre la conjura y el conjuro, entre la solidaridad y la magia. Es sabido que Guibert de Nogent, en su De vita sua, llamaba al juramento de apoyo mutuo (pacto por el que los siervos mutualizaban o colectivizaban sus obligaciones para aliviar las cargas señoriales) mutui adjutorii conjuratio: «a new and detestable word. Through it the serfs (capite censi) are freed from all serfdom» (Kropotkin 1902: 178). No solamente, pues, se estigmatiza la reivindicación del valor añadido inherente al trabajo reproductivo, sino también y, sobre todo, la repentina communitas femenina y masculina que este trabajo generaba y que engrasaba desde

7 Como apunta Broedel, con el Malleus no solo se justifica la brujería, sino que se implementa una ofensiva contra las mujeres: «Institoris and Sprenger's innovation was not their insistence that women were naturally prone to practice maleficium [...] Rather, it was their claim that harmful magic belonged exclusively to women that was new» (2003: 175). 
dentro el modo de producción feudal, sentando a la vez las condiciones básicas de su propia negación.

En La Celestina, los momentos que rememoran y que fugazmente actualizan esta comunidad perdida son más que significativos. Asociados, more bajtiniano, al sexo y a la comida, tienen lugar en casa de Celestina y congregan a una servidumbre alborozada que comparte lo poco que tiene en un clima de convivialidad indiferente a la edad, linaje o género de sus participantes. A su alrededor, y mientras este clima prevalece, todo lo que puede disfrutarse en común es común. Por eso Celestina saqueaba tumbas (huesos que no son de nadie, huesos que son de todos) en compañía de su amiga Claudina y, por eso, el robo de seis pares de pollos para pertrechar las despensas del banquete de celebración [«comamos y holguemos, que nuestro amo ayunará por todos» (Rojas 2011: 137)] es llevado a cabo por Pármeno con toda naturalidad. Me refiero al famoso banquete del acto noveno, que debe ser catalogado como un auténtico aquelarre - auténtico porque los aquelarres de «brujas» son la metáfora de estas pequeñas conjuras cotidianas y no al revés - en el sentido que Federici imprime a esta palabra.

Tratemos de imaginar este banquete en un entorno rural. En el páramo urbano de las nuevas relaciones de producción, dispersas y fraccionadas, puede resultar tan anecdótico como una pintoresca escena de costumbres, pero en el marco de las relaciones asociativas de una villa feudal, donde las familias requieren de otras familias para producir y subsistir, debería haber encendido las alarmas de ese señor ausente que Rojas trata de restituir devolviendo la potestad del castigo al Señor que está en los cielos. Elicia y Areúsa no dejan de desprestigiar a Melibea y ponderar los abusos de sus amas, tal y como expresa Areúsa:

¡Oh tía, y qué duro nombre y qué grave y soberbio es «señora» contino en la boca! Por esto me vivo sobre mí desde que me sé conoscer. Que jamás me precié de llamarme de otro sino mía [...] Nunca oyen [las criadas] su nombre propio de la boca de ellas; sino 'puta' acá, 'puta' acullá (Rojas 2011: 152).

Lucrecia, como nota Lina Ruiz Guzmán, «también aparece para completar este complot: lleva el mensaje de Melibea y escucha con nostalgia el "alegre tiempo" del que habla Celestina» (21). Tanto es así que no puede evitar decir: «así me estuviera un año sin comer, escuchándote y pensando en aquella vida buena» (Rojas 2011: 155). Este recuerdo todavía palpitante de las relaciones de horizontalidad que vertebraban la institución del servicio en el mundo feudal (y que igualaban a ayos y lacayos, «labranderas» y labradoras, mozos y viejas, amigos y hermanos, hombres y mujeres) es la dimensión más soslayada de la obra, si no su propio modo de latir. Dicho de otra manera: lo que esta historia nos está contando, bajo el barniz dignificante del affair entre Calisto y Melibea, es la descomposición 
de estas relaciones «improductivas» con la llegada y el triunfo de las relaciones salariales; improductivas, claro está, desde el punto de vista de la nueva ideología burguesa, puesto que en la aldea feudal eran totalmente productivas y necesarias. Ninguna frase, aunque hay muchas que podrían hacerlo, resume mejor esta descomposición que la claudicación del hijo de la Claudina en el doceno auto, cuando finalmente llega a la conclusión de que «sobre dinero no hay amistad» (Rojas 2011: 190) o, lo que es lo mismo, de que donde hay dinero no hay compañero. Y ningún lamento atraviesa tan profundamente el corazón de la obra que el que espeta el pobre Sosia en el acto treceno al enterarse de que Pármeno y Sempronio han sido decapitados. Los han matado, llora, a «nuestros compañeros, nuestros hermanos...»(Rojas 2011: 200).

Nuestros compañeros, nuestros hermanos. El presente también es historia (solo para un historicismo de anticuario deja de serlo) y la reacción de un lector actual revela mejor que ninguna otra cosa las costuras de la obra, invisibles para unos personajes que sienten el tejido que ciñe sus miembros como una segunda piel. Los estudiantes estadounidenses que leen la obra de Rojas por primera vez asisten sorprendidos al trato extrañamente familiar que se dispensan estos personajes. Los más despistados incluso llegan a confundir a Pármeno y Sempronio con los hijos de Celestina, al calor de lo que perciben como una asombrosa consanguineidad sin lazos consanguíneos. ¡Cómo no hacerlo, si cuando Celestina se dirige a Pármeno [«¿Ríeste, landrecilla, hijo?» (43)] lo interpela maternalmente, y cuando Pármeno responde [«Calla, madre, no me culpes ni me tengas, aunque mozo, por insipiente» (43)] sella el parlamento con un sincero cariño filial! Madres, hijos, hermanos, primos y sobrinos se permutan en este recordatorio de la gran familia extendida de la aldea feudal, que la guerra de intereses individuales del mundo capitalista llevará a la quiebra ${ }^{8}$. Esta es la historia que se nos ha contado y esta es la que

$\overline{8}$ Los ejemplos de la persistencia de esta gran familia extendida, que existía desde el llamado feudalismo carolingio, abruman al lector: «Dile que viene tu primo y mi familiar», avisa Celestina a Elicia para que esconda a Crito de su amante; «Pármeno hijo, después de las pasadas razones no he habido oportuno tiempo para te decir y mostrar el mucho amor que te tengo», le dice la vieja al muchacho (al que genuinamente quiere) al comienzo del séptimo auto; «Pármeno, hermano», llama Sempronio a su amigo y viceversa, casi cada vez que el uno se dirige al otro (Rojas 2011: 33, 115 y 134). Cavallo explica que en la Edad Media las familias eran grupos interfamiliares extendidos: «the tendency for both spouses, or at least for the wife, to marry when very young meant that the new couple often lived with the husband's family in the early stages of marriage. A woman could give birth to all her children $[\ldots]$ before having her own household» (2012: 27). En La Celestina se observa esta familia extendida (Areúsa y Elicia son primas. Lucrecia es prima de Elicia y Pármeno es hijo de Claudina, a quien Celestina ha criado como su hijo, etc.), pero también los que no son parientes se comportan con los demás como si lo fueran, de acuerdo, por cierto, a lo que Marx llamaba forma expandida del valor (estadio previo a la forma general del valor en las economías dinerarias). 
no se nos ha mostrado todavía: no tanto el relato de la irrupción de una economía simbólica capitalista que viene a negar el habitus feudal (e.g., la lectura canónica de José Antonio Maravall), sino la negación, por parte del modo de producción feudal, de sus tendencias inherentes de cambio, cuya síntesis - negación de una negación- constituye el modo de producción capitalista. Tal precisión teórica supone, en términos prácticos, la diferencia entre poner el acento en la sacrosanta «individualidad» de los personajes (considerada por Maravall como algo estéticamente bueno, algo que los hace redondos, humanos, de carne y hueso, etc.) y enfatizar su carácter comunitario, su pertenencia a esa gran familia extendida que no entiende de parentescos (Maravall 1968: 14-17). En otras palabras: los personajes de La Celestina no son mejores por ser más individuales, sino por serlo cualitativamente menos, enredados como están en la familia sin familia de la communitas medieval.

Y es que, bajo el esmalte ideológico de un discurso todavía feudal, asoman constantemente los mimbres rotos de una comunidad desplazada, de la que banquetes, carnavales y cónclaves vecinales constituyen apenas una reconstrucción pasajera y un testimonio fúnebre. Una vez más, las palabras de Areúsa son claras a propósito de qué echa de menos una mujer del común y qué le permite recuperar el ejercicio precario de la prostitución:

Que estas que sirven a señoras ni gozan deleite ni conoscen los dulces premios del amor. Nunca tratan con parientas ni con iguales a quien pueden hablar tú por tú, con quien digan: «¿qué cenaste?», «¿estás preñada?», «¿cuántas gallinas crías?», «llévame a merendar a tu casa»; «imuéstrame tu enamorado!», «¿cuánto ha que no te vido?», «¿cómo te va con él?», «¿quién son tus vecinas?», y otras cosas de igualdad semejante (Rojas 2011: 151-152).

No es precisamente el dinero o la sed de autonomía lo que mueve a Areúsa, como una lectura liberalizante de la obra nos empujaría a presuponer casi de manera refleja, sino la nostalgia de una comunidad igualitaria que todavía sobrevivía en la aldea feudal y que se resquebraja en el momento en el que las aldeanas entran a servir a sus nuevas amas en las casas de alcurnia de la ciudad.

La lectura de Federici, o una lectura desde Federici, nos permite finalmente ubicar el papel de Celestina como hechicera (el papel de las mujeres, al fin y al cabo) en este drama. No en vano, desestimar la importancia de las dotes hechiceriles de Celestina y de su importancia en la obra en favor del sempiterno libre albedrío de Melibea significa volver a desvalorizar, más de quinientos años después, el trabajo reproductivo y supuestamente improductivo de Celestina, que es parte de un trabajo común o un trabajo de lo común. Además, la lectura desde Federici nos permite acceder a esa otra «Edad Media» que la lectura liberal-humanista está, disimuladamente o sin querer, barriendo debajo de la alfombra, lectura para la que la brujería y su represión serían solamente vestigios de un remanente católico-feudal 
exclusivamente patrocinado por la santa Inquisición?. Es cierto que la Inquisición, que, por cierto, no tenía jurisdicción en toda la península, sí fue particularmente virulenta en lugares como Aragón a finales del siglo xv. Pero la realidad es que muchos de los juicios sumarísimos fueron llevados a cabo por tribunales seculares y que la caza de brujas contribuyó más a cicatrizar las diferencias entre la Europa católica y la Europa protestante que a profundizar en su división. Federici documenta con robusta evidencia que el problema de la brujería no es un problema «medieval», sino un problema que tiene que ver con los ajustes que el primer capitalismo necesita hacer para consumar ese proceso en marcha que Marx llamó acumulación primitiva. También es verdad, como Federici misma nota, que entre los siglos VII y VIII el delito de maleficium estaba tipificado en el derecho teutónico, pero solo para justificar la criminalización de los infieles sarracenos (Federici 2010: 224-25). Solo a partir de mediados del siglo xv comienza la persecución a gran escala, coincidiendo con la aparición de los primeros documentos que contienen descripciones de aquelarres. Entre 1435 y 1487 se escribieron nada menos que veintiocho tratados sobre brujería. De fecha muy cercana a la publicación de la obra de Rojas, 1486, data el tristemente célebre Malleus maleficarum. La obra de Heinrich Kramer solo seguía la estela de una reciente bula papal, la Summis desiderantes de Inocencio VIII (1484), que señalaba a la brujería femenina como

$9 \quad$ Por supuesto, con excepciones notables. Ya José Antonio Maravall (1968) estudiaba cómo el «individualismo» había dado al traste con un mundo que le era ajeno y, aunque nunca llegara a clarificar (mucho menos a teorizar) cuál era la base de esa nostalgia que rezuma por todos los poros de la obra, la destrucción de la comunidad feudal estaba, como si dijéramos, injerta en su argumentación. Lamentablemente, con todo, Maravall no entiende la importancia de la communitas en La Celestina. Así, a la hora de evaluar uno de esos efluvios de nostalgia en boca de Pármeno (que a su vez está citando algo que le ha oído decir a Celestina), Maravall se pega un llamativo traspiés. Pármeno dice en el octavo auto: «de ninguna prosperidad es buena la posesión sin compañía. El placer no comunicado no es placer» (Rojas 2011: 134). Y todo lo que Maravall puede extraer de la cita de Séneca es otra muestra de esa ostentación señorial a la que, para él, se reducen las actitudes de todos los personajes de la tragicomedia; en este caso, el placer de ostentar de Pármeno, que mimetiza el de su señor Calisto: «la ostentación, esto es, la manifestación pública o social de todo lo bueno y rico que se posee o de que se goza, es ley en el mundo de La Celestina» (Maravall 1968: 35). La simple reivindicación del gozo de compartir es invisible dentro de este (erróneo) protocolo de lectura. Otro tanto puede decirse del brillante artículo de Raúl Álvarez Moreno (2011). Álvarez Moreno consigue entender como pocos que «los conflictos, inseguridad y caos, de los que la estructura dramática e ideológica de Celestina participa junto a la sociedad castellana, remiten últimamente a la propiedad; en concreto, a cómo se obtienen y para qué se usan los bienes exteriores dentro del código señorial mencionado. Estos dos ejes [son] adquisición/ disposición y bien particular/bien común» (14). Sin embargo, cuando se trata de valorar el recuerdo de una edad de oro (ensoñación que comparten Celestina y su prole), Álvarez Moreno solo es capaz de remitirse a la bonanza económica de una Celestina más joven. Asimismo, en fórmulas como el «todo lo mío es tuyo» de Celestina, Álvarez no ve los ecos de una propuesta que tuvo y ha dejado de tener sentido, sino un mero intento de manipulación para ganarse la voluntad de sus secuaces. 
una pujante amenaza. Y esto no dejaba a España fuera del tablero. En 1498, por ejemplo, la Inquisición zaragozana condenó a la hoguera a Gracia del Valle, como documenta Eva Lara Alberola. Le seguiría en 1499 (fecha de publicación de La Celestina) María, mujer de García Bielsa. También a finales del siglo Xv se dan los primeros casos documentados de brujería en el País Vasco, que se multiplicarían en los años subsiguientes cobrándose decenas de vidas, como las de las veintinueve mujeres navarras que fueron condenadas a muerte en 1507 (Lara Alberola 2010: 93). La caza de brujas alcanzó su cénit entre 1580 y 1630, es decir, en la época en la que las relaciones feudales ya estaban dando paso a las instituciones económicas y políticas propias del capitalismo mercantil. La Constitutio criminalis Carolina -el código legal Imperial promulgado por Carlos V en 1532- establecía que la brujería sería penada con la muerte; hablamos, naturalmente, de un código civil diseñado para garantizar la salud de la república en el contexto del mercantilismo, no de un catecismo religioso. Federici se sacude de esta manera otro de los mitos intemporales de la vulgata crítica liberal, el que consiste en culpar de todo a la superstición feudal, a la religión católica y a la Inquisición con el fin de vindicar una mayor dosis de laicismo y de beatífico libre mercado.

Esto no significa, claro está, que existiera una campaña orquestada por parte de los poderes económicos y políticos para garantizar la acumulación de plusvalías a partir de la devaluación de la reproducción de la fuerza de trabajo, al menos no como agenda explícitamente articulada en los términos de una racionalidad instrumental capitalista. Por el contrario, lo que encontraremos es una articulación ideológicamente feudal de esta empresa, donde la conceptualización de estas mujeres como brujas y, por tanto, como adoradoras del demonio, solo evidencia la persistencia de los binomios «servicio bueno/servicio malo» $\mathrm{y}$ «señor bueno/señor malo», a través de los cuales y solo a través de los cuales el engranaje de la acumulación se puede poner en marcha. Esta es quizá la mayor debilidad del análisis de Federici: su incapacidad de conjugar el desfase entre el nivel político-económico del análisis y el nivel ideológico del mismo, que requeriría un retorno al Marx de Althusser y a su concepto de desarrollo impar (1997: 312). Su tesis a propósito de la relación entre la brujería y el régimen de acumulación primitiva, sigue siendo, sin embargo, absolutamente válida: más del $80 \%$ de las personas juzgadas y ejecutadas en Europa en los siglos XVI y XVII por el crimen de brujería fueron mujeres. De hecho, fueron perseguidas más mujeres por brujería en este periodo que por cualquier otro crimen, excepto, significativamente, el de infanticidio. Esto prueba que la herejía en sí misma no constituye una explicación suficiente. Otra diferencia entre las meras persecuciones de herejes y las de las brujas en el siglo XVI es que las acusaciones de perversión sexual e infanticidio contra las brujas tenían un papel central y estaban acompañadas por la virtual demonización de las prácticas anticonceptivas, o de las hierbas y ungüentos que se procuraban con tal fin y 
que recibían el previsible nombre de maleficia $^{10}$. Significativamente, o poco significativamente a estas alturas de este trabajo, la elaboración de estos maleficios es una de las especialidades de Celestina, como confirma la irrepetible visita guiada que Pármeno nos brinda a su curiosa rebotica en el primer auto (Rojas 2011: 38-39).

Sorprende, en este sentido, la omisión generalizada del trabajo de Federici en la bibliografía celestinesca, mucho más todavía a la luz de la enorme resonancia internacional de que este trabajo, traducido a varios idiomas, ha gozado en otras áreas disciplinarias ${ }^{11}$. Lo que sin ánimo de herir ninguna susceptibilidad me he permitido llamar «el escándalo de Celestina» se robustece, de hecho, sobre la base de este silencio, por desgracia habitual en cierto hispanismo que, con afortunadas y honrosas excepciones, tiende a vivir de espaldas a los debates que dominan en otros campos de las ciencias humanas y sociales. El citado libro de Lara Alberola es un ejemplo de ello. La obra, que exhibe un loable y necesario despliegue de medios de archivo, repasa las fuentes lexicográficas, rastrea los antecedentes del problema en el pensamiento grecorromano e incluso desarrolla una taxonomía de tipos de hechiceras, además de proporcionar datos valiosos extraídos de abundante documentación jurídica y curial. A lo largo de este recorrido, Lara Alberola llega a conclusiones similares a las que Federici había arribado en la década anterior, confirmando que el caso español no fue, a grandes rasgos, una excepción con respecto a la norma europea en lo que toca a la gestión penal y social de la brujería. Lara Alberola destaca, como Federici, que la polémica en torno a la brujería no es un fenómeno del medioevo, sino que comienza a manifestarse en la literatura legal a partir del siglo xv:

Este es el panorama del derecho medieval en cuanto a hechicería. No se hace apenas alusión a las mujeres como portadoras del mal. Dicha concepción se va desarrollando paulatinamente y se manifestará a partir del siglo XII, pero, sobre todo, en los siglos XV-XVII. Estos testimonios demuestran la no existencia de la brujería hasta bien avanzado el Medioevo y el hecho de que dicho fenómeno es totalmente moderno (2010: 90).

Y, asimismo, sanciona el hecho de que la persecución de la brujería está ligada al control de las funciones reproductivas, acudiendo a testimonios como el siguiente fragmento del tercer folio de los Estatutos y desafueros contra las hechizeras y bruxas, hechos y otorgados por los Jurados y Concejo General de la villa

\footnotetext{
10 Sobre la relación entre contracepción, aborto y brujería en el mundo feudal (Sauer 2015: 32-34).

11 Apenas he podido encontrar dos puntualísimas menciones en el campo del hispanismo, ambas muy recientes (2016) y anteriores a la primera redacción de este artículo. Una es la de Contreras Elvira (2016: 46); la otra, mucho más importante por lo que a la aportación de su trabajo se refiere, es la de Zamora Calvo (2016: 65). Ninguna de ellas, sin embargo, tiene una relación directa con la crítica celestinesca.
} 
y lugares del justiciado de Gia, que data de 1592 y que se conserva en el Archivo Diocesano de Barbastro (Huesca):

Han muerto, hecho matar o morir faran personas grandes o pequeñas, ganados gruesos o menudos [...] han ligado o ligaran o ligar faran a qualesquiere personas [...] han impedido, impidirán o hecho impidir que marido y mujer carnalmente se puedan conoçer o que alguna mujer no se pueda preñar, o los partos de las mugeres han dampnifficado o dampnifficaran (Lara Alberola 2010: 92).

Sin embargo, el trabajo de Federici no aparece mencionado ni una sola vez en la generosa bibliografía que jalona las 367 páginas del libro de Lara Alberola. El descuido no carece de trascendencia, pues priva a la autora de la posibilidad de ofrecer una explicación convincente acerca de las razones concretas por las que la brujería irrumpe en España en este momento histórico. En su lugar encontraremos explicaciones claramente vagas e insatisfactorias, que atribuyen a «luchas en diferentes frentes: religioso, social e ideológico», no menos que a otros «factores» como «las guerras, las epidemias [o] los levantamientos del siglo», la necesidad de encontrar «chivos expiatorios como las brujas» (2010: 84). Lara Alberola no explica cuáles son concretamente estas luchas, ni por qué, en concreto, las brujas se convierten en chivos expiatorios. Por lo demás, la constelación empirista de factores o «confluencia de causas» a la que Lara Alberola apela para dar cuenta de la obsesión por lo diabólico ya desde el siglo XIV (recuperación de la cultura antigua, reorganización del poder eclesiástico, crisis económica) olvida un hecho fundamental que está más allá de los hechos crudos: ofrecer una explicación histórica significa precisamente estructurar y ordenar una confluencia de causas dada dentro de un marco teórico (2010: 64). Esto es lo que la propuesta de Federici ofrecía en su viaje de Marx a Foucault (del trabajo al biotrabajo) y esto es lo que permanece ausente del estado de la cuestión en lo que concierne al tema de la brujería en La Celestina. Al final, quizá, de lo que se trataba era de prestar más atención a esas «luchas» sobre las que Lara Alberola, no menos que buena parte de la crítica, pasa de puntillas; las luchas, contradicciones o conflictos que arriba nos atrevíamos a describir como una dialéctica. No en vano, el propio Rojas ya nos avisaba en el prólogo a su obra de que todas las cosas son «criadas a manera de contienda o batalla» (Rojas 2011: 15). Por supuesto, Rojas no está hablando ni tiene por qué estar hablando de la lucha de clases tal y como Marx desarrollaría este concepto en El capital, pero tampoco Heráclito de Éfeso estaba pensando en el mundo en que La Celestina tendría lugar cuando escribió la sentencia que Rojas cita. Sea como fuere, la realidad de la obra no cambia. La otra, lamentablemente, tampoco. 


\section{BIBLIOGRAFÍA}

Alfonso X «El SABio» (1807). Las siete partidas del Rey Don Alfonso el Sabio. Madrid: Imprenta Real.

Althusser, Louis and Étienne BaliBar (1997). Reading Capital. London/New York: Verso.

Álvarez Moreno, Raúl (2011). «Propiedad y dominium en Castilla a finales del siglo xv: Celestina como civitas non recte instituta». Celestinesca, 35, pp. 9-42.

Botta, Patrizia (1994). «La magia en La Celestina». Dicenda. Cuadernos de Filología Hispánica, 12, pp. 37-67.

BrinkoP, Claudia (2014). «De mujer sabia a mulier maléfica: el cuerpo pasional en la hoguera de la modernidad». En Marina Fe (coord.), Mujeres en la hoguera: Representaciones culturales y literarias de la figura de la bruja. Ciudad de México: Universidad Nacional Autónoma de México, pp. 41-51.

Broedel, Hans Peter (2003). The Malleus Maleficarum and the Construction of Witchcraft: Theology and Popular Belief. Manchester: Manchester University Press.

Canet Vallés, José Luis (2000). «Hechicería versus libre albedrío en La Celestina». En Juan Carlos Elorza Guinea (ed.), El jardin de Melibea. Madrid: Sociedad Estatal para la Conmemoración de los Centenarios de Felipe II y Carlos V, pp. 201-211.

CÁrdenas-Rotunno, Anthony (2001). «El pacto diabólico en La Celestina». En Felipe B. Pedraza et alii (eds.), La Celestina: V Centenario (1499-1999). Actas del Congreso Internacional. Cuenca: Universidad de Castilla-La Mancha, pp. 369-376.

Carmona Ruiz, María Antonia (1995). Usurpaciones de tierras y derechos comunales en Sevilla y su «tierra» durante el siglo XV. Sevilla: Ministerio de Agricultura.

Cavallo, Sandra (2012). «Family Relationship». En Sandra Cavallo and Silvia Evangelisti (eds.), A Cultural History of Childhood and Family in the Early Modern Age. London: Bloomsbury Academic, pp. 5-32.

Contreras Elvira, Ana (2016). «La transmisión de la Alta Magia renacentista en el teatro popular del siglo XVIII: La comedia de magia como teatro de la memoria y libro de invocaciones». En María Luisa Lobato, Javier San José y Germán Vega (eds.), Brujería, magia y otros prodigios en la literatura española del Siglo de Oro. Alicante: Biblioteca Virtual Miguel de Cervantes, pp. 45-86.

DANGler, Jean (2001). Mediating Fictions: Literature, Women. Healers, and the Go-Between in Medieval and Early Modem Iberia. Lewisburg, PA: Bucknell University Press.

FEDERICI, Silvia (2010). Calibán y la bruja. Mujeres, cuerpo y acumulación originaria. Verónica Hendel y Leopoldo Sebastián Touza (trads). Madrid: Traficantes de Sueños.

GuAzzo, Francesco Maria (1988). Compendium maleficarum: The Montague Summers Edition. E. A. Ashwin (ed.). New York: Dover.

Herrero, María del Carmen y Cristina Pérez Galán (coords.) (2014). Mujeres de la Edad Media: actividades políticas, socioeconómicas y culturales. Zaragoza: Institución Fernando «el Católico».

Institoris, Heinrich and Jakob Sprenger (1970). Malleus Maleficarum. New York: Benjamin Blom. 
JAMESON, Fredric (1973). «The Vanishing Mediator: Narrative Structure in Max Weber». New German Critique, 1, pp. 52-89.

Kropotkin, Pëtr (1902). Mutual Aid: A Factor of Evolution. New York: McCLure Phillips $\&$ Co.

Lara Alberola, Eva (2010). Hechiceras y brujas en la literatura española de los Siglos de Oro. València: Publicacions de la Universitat de València.

Lara Alberola, Eva y Alberto Montaner (2016). «La hechicería en La Celestina desde el estudio de la magia». En Emilio Blanco (ed.), Grandes y pequeños de la literatura medieval y Renacentista. Salamanca: SEMyR, pp. 447-449.

Le Franc, Martin (1485). Le Champion des dames. Lyon: Guillaume le Roy.

López Griguera, Luisa (2005). «Causas de las acciones de los personajes». En Ottavio Di Camillo and John O'Neill (eds.), Selected Papers from the International Congress in Commemoration of the Quincentennial Anniversary of La Celestina. New York: Hispanic Seminary of Medieval Studies, pp. 111-124.

Lozano Renieblas, Isabel (2005). «La Celestina en el contexto de los pactos demónicos». En Ottavio Di Camillo and John O'Neill (eds.), Selected Papers from the International Congress in Commemoration of the Quincentennial Anniversary of $\mathrm{La}$ Celestina. New York: Hispanic Seminary of Medieval Studies, pp. 153-164.

Maravall, José Antonio (1968). El mundo social de "La Celestina”. Madrid: Gredos.

Marcos Martín, Alberto (1997). «Evolución de la propiedad pública municipal en Castilla la Vieja durante la época moderna». Studia Historica. Historia Moderna, 16, pp. 57-100.

Martín Martín, José Luis (1990). «Evolución de los bienes comunales en el siglo XV». Studia Historica. Historia Medieval, 8, pp. 7-46.

Marx, Karl (2007). Capital: A Critique of Political Economy. New York: Cosimo, t. 1

Muñoz Fernández, Ángela y Cristina Segura Graíño (coords.) (1988). El trabajo de las mujeres en la Edad Media hispana. Madrid: Regesta Imperii.

Padilla Carmona, Carles (2017). «Precedentes clásicos del conjuro del acto tercero de $L a$ Celestina». eHumanista, 36, pp. 231-240.

Pérez Priego, Miguel Ángel (2000). «El conjuro de Celestina». En Pilar Carrasco Cantos (coord.), El mundo como contienda: estudios sobre La Celestina. Málaga: Universidad de Málaga, pp. 77-88.

PuEYo, Víctor (2012). «Sobre la categoría del mediador evanescente: pícaros, bufones y prostitutas en el umbral de la modernidad». Journal of Spanish Cultural Studies, 13.2, pp. 149-65.

Rider, Catherine (2006). Magic and Impotence in the Middle Ages. Oxford: Oxford University Press.

RoJAs, Fernando de (2011). La Celestina. Francisco Lobera y Guillermo Serés (eds.). Madrid: Real Academia Española.

Ruiz Guzmán, Lina. «La Celestina: poder y exclusión de las mujeres en la era del mercantilismo» (texto inédito).

Russell, Peter (1978). Temas de La Celestina y otros estudios. Barcelona: Ariel. 
SAUER, Michelle M. (2015). Gender in Medieval Culture. London: Bloomsbury Academic. Severin, Dorothy Sherman (1993). «Celestina and the Magical Empowerment of Women». Celestinesca, 17.2, pp. 11-28.

SEverin, Dorothy Sherman (1995). Witchcraft in Celestina. London: Queen Mary and Wesfield College.

Severin, Dorothy Sherman (2006). «Two Fifteenth-Century Spanish Literary Conjurations and their Relationship to Lucan's Pharsalia, VI». En Charles Burnett, Jill Kraye y W. F. Ryan (eds.), Magic and the Classical Tradition. London: Warburg Institute, pp. 213-222.

SEVERIN, Dorothy Sherman (2007). «Witchcraft in Celestina: A Bibliographic Update since 1995». La corónica: A Journal of Medieval Hispanic Languages, Literatures, and Cultures, 36.1, pp. 237-243.

Solomon, Michael (1997). «Women Healers and the Power to disease in Late Medieval Spain». En Lilian R. Furst (ed.), Women Healers and Physicians: Climbing a Long Hill. Lexington: Kentucky University Press, pp. 79-92.

Zamora Calvo, María Jesús (2016). Artes maleficorum. Brujas, magos y demonios en el Siglo de Oro. Barcelona: Calambur Editorial.

ŽıžEK, Slavoj (2008). For They Know Not What They Do: Enjoyment as a Political Factor. London: Verso.

Recibido: $17 / 03 / 2019$

Aceptado: 03/05/2019 


\section{wa}

El escándalo de La CELESTINa: Magia y aCumUlación PRIMitiva EN LA EsPaña DEl holocausto (1486-1507)

RESUMEN: Este artículo examina el estado de la cuestión sobre la magia y la brujería en La Celestina. Lo hace centrándose en un muy sintomático silencio: el silencio alrededor de la intervención seminal de Silvia Federici sobre el asunto en su ya clásico Calibán y la bruja, silencio que en mi opinión no desmerece el calificativo de escandaloso. Sostengo que la obra maestra de Fernando de Rojas no puede ser propiamente evaluada sin prestar completa y escrupulosa atención a los complejos procesos históricos que subyacen a las dinámicas de caza de brujas en la baja Edad Media. La caza de brujas no es un fenómeno religioso o incluso un fenómeno político, o (para ser más precisos) es un fenómeno religioso y un fenómeno político solo en la medida en que participa del más amplio panorama de la acumulación primitiva durante la transición del modo de producción feudal al modo de producción capitalista. Culpando obsesivamente a la Iglesia católica y a sus aparatos represivos (i.e., la Inquisición), la crítica liberal menoscaba este simple hecho. En mi cuestionamiento de esta tendencia hermenéutica, originalmente apadrinada por José Antonio Maravall en los sesenta y seguramente hegemónica hoy, muestro cómo La Celestina no puede entenderse sino como un testimonio nostálgico de la no tan lejana economía medieval del procomún.

Palabras clave: Celestina, brujería, Inquisición, Federici, privatización, acumulación primitiva, procomún.

\section{Celestina's SCANDAL: MAGIC AND PRIMITIVE ACCUMULATION IN Holocaust SPAIN (1486-1507)}

ABSTRACT: This article examines the state of the question on magic and witchcraft in La Celestina. It does so by focusing on a very symptomatic silence: the silence around Silvia Federici's seminal intervention on the matter in her already classic Caliban and the Witch, which in my view is nothing short of a scandal. I argue that Fernando de Rojas' masterpiece cannot be properly assessed without paying full and thorough attention to the complex historical processes underlying the dynamics of witch-hunting in late-medieval Europe. Witch-hunting is not a religious or even a political phenomenon, or (to be more precise) it is a religious and a political phenomenon only insofar as it partakes in the broader economic landscape of primitive accumulation during the transition from the feudal to the capitalist mode of production. By obsessively putting all the blame on the Catholic Church and its repressive apparatuses (i.e., the Inquisition), liberal criticism is underscoring this very simple fact. In my critique to this critical trend, originally espoused by José Antonio Maravall in the 60s and arguably hegemonic today, I show how La Celestina cannot but be understood as a nostalgic testimony for the not-so-distant economy of the medieval commons.

KeYwords: Celestina, witchcraft, Inquisition, Federici, privatization, primitive accumulation, the commons. 